\title{
CORRELACIÓN Y COMPONENTES PRINCIPALES DE VARIACIÓN EN VARIEDADES DE PAPA (Solanum tuberosum L.) ${ }^{1}$
}

\author{
Fernando Borrego E..$^{2}$, José M. Fernández B.3., Alfonso López B.2, Víctor M. Parga T.4, \\ Margarita Murillo S. ${ }^{2}$, Adrián Carvajal A. ${ }^{5}$
}

\begin{abstract}
RESUMEN
Correlación y componentes principales de variación en variedades de papa (Solanum tuberosum L.). Se evaluaron siete genotipos de papa en campo, en un diseño de bloques al azar con cuatro repeticiones, la parcela experimental de tres surcos de cinco metros de largo. Las variables analizadas fueron morfológicas, fisiológicas, agroclimáticas y de rendimiento. Se determinaron correlaciones simples y análisis de componentes principales (ACP), para encontrar el grado de asociación entre variables, y cuales fueron las variables que más contribuyeron a la variación en el rendimiento. Las variables más correlacionadas con rendimiento fueron altura de planta, peso seco de hojas y contenido de clorofila. El ACP permitió una mejor discriminación entre variables; las más importantes fueron la fotosíntesis y uso eficiente del agua. Las mejores variedades al considerar todas las variables en conjunto, fueron Norteña, Mondial y Atlantic, superando al testigo regional (Alpha).
\end{abstract}

\begin{abstract}
Correlation and main components of variation in varieties of potato (Solanum tuberosum L.). Seven potato genotypes were evaluated on field, with a random block design with four repetitions, and an experimental plot of three rows measuring five $\mathrm{m}$ long. The variables analyzed were morphological, physiological, agroclimatic and yield, by calculating Correlations and Principal Components Analysis (PCA), in attempt to find the degree of association between variables, and which variables contributed to the variation in the yield. The most correlated variables in the yield were plant height, the dry weight of leaves, and chlorophyll content. The PCA permitted a better screening between variables, such as photosynthesis, water use efficiency. When considering all variables together, the best varieties were Norteña, Mondial and Atlantic, found to be superior to the regional genotype (Alpha).
\end{abstract}

\section{INTRODUCCIÓN}

El rendimiento económico de un cultivo es la resultante de integrar a lo largo de la ontogenia del vegetal, los procesos fisiológicos de asimilación y mantenimiento que permiten el crecimiento y acumulación de fotosintetizados, en un ambiente de crecimiento determinado. Puede decirse que es una transformación de energía llevada principalmente por tres procesos secuenciales a) intercepción, por el follaje, de la radiación solar incidente, b) conversión de la energía radiante interceptada, a energía química potencial (biomasa) y c) partición de la biomasa entre las partes cosechables y el resto de la planta (Hay y Walker, 1989).

Dada la cantidad de variables morfológicas y fisiológicas que intervienen en el rendimiento, el fitomejorador toma en cuenta principalmente el rendimiento y algunos de sus componentes visuales más importantes, a los cuales puede dar una calificación fenotípica, para avanzar rápidamente en la evaluación de genotipos. Sin embargo, muchas veces toma decisiones equivocadas con este procedimiento simplista y puede descartar genotipos valiosos. Se ha propuesto el uso de Análisis de

\footnotetext{
1 Parte de Tesis de Grado de Doctorado, Universidad Autónoma Agraria “Antonio Narro”. México.

2 Dpto. de Fitomejoramiento. Universidad Autónoma Agraria “Antonio Narro”. Buenavista, Saltillo, Coahuila, México, 25315.

3 Dpto. Botánica, UAAAN.

4 INIFAP-CESIA, Saltillo, Coahuila, México. 25090.

5 Dpto. Fitomejoramiento, UAAAN.
} 
componentes principal (ACP) para seleccionar con mayor precisión genotipos a introducir (Farías, 1983) y que, comparados con otros métodos multivariados (Indices de Selección, Análisis de Factores) da mejores resultados, permitiendo un ahorro de recursos físicos y de tiempo, dando al fitomejorador más flexibilidad en determinar el número de familias y el tamaño de familia a ser evaluada, así como determinar la combinación de características que constituyen una planta "ideal" (Goldshalk y Timothy, 1988), así como también por el estudio de las correlaciones simples.

El (ACP) presenta múltiples ventajas (Broschat, 1979): es una técnica que reduce la dimensionalidad de un conjunto de datos multivariados, remueve las interrelaciones existentes entre variables, organiza los datos en forma de vectores ortogonales en donde cada una de las variables dentro del vector se comporta en forma similar en base a sus correlaciones; a cada uno de estos vectores se le llama componente principal. Esta prueba también nos expresa la mayor parte de la varianza de los datos ortogonales, y determina que esta prueba es una herramienta útil para simplificar el análisis e interpretación de la gran cantidad de variables consideradas en una evaluación exhaustiva.

Para realizar el presente trabajo se establecieron los siguientes objetivos: a) determinar las variables morfológicas, fisiológicas y agroclimáticas, de mayor relación con el rendimiento y b) determinar los mejores genotipos considerando todas las variables en conjunto.

\section{MATERIALES Y MÉTODOS}

Localización del área de estudio: El experimento de campo se realizó en el ciclo agrícola Verano-Otoño de 1996, en el campo experimental de la Universidad Autónoma Agraria "Antonio Narro" (UAAAN) en Buenavista, Saltillo, Coahuila, y en el laboratorio de Fisiotecnia de la misma Universidad. Las características principales del área son: $25^{\circ} 22^{\prime}$ latitud N, $101^{\circ} 03^{\prime}$ longitud $\mathrm{W}$, altitud $1743 \mathrm{msnm}$. La temperatura media anual es de $19,8^{\circ} \mathrm{C}$. Los meses más cálidos son junio, julio y agosto, con temperaturas que alcanzan hasta $39^{\circ} \mathrm{C}$, mientras que en los meses de diciembre y enero, se registran las temperaturas más bajas, de hasta $-13^{\circ} \mathrm{C}$, presentándose heladas regulares en el período de noviembre a marzo. La precipitación media anual es de 350 a $450 \mathrm{~mm}$, siendo los meses más lluviosos julio, agosto y septiembre; en la época de invierno, las lluvias que se presentan son moderadas. Tipo de clima : BWhw $\left(x^{\prime}\right)(e)$ : clima muy seco, semicálido, con invierno fresco, con lluvias de verano y precipitación invernal al
$10 \%$ del total anual. El fotoperíodo medio anual es de 11,99 horas.

Variedades: Alpha, Mondial, Norteña, Gigant, Atlantic, Snowden y Russet Burbank. Soto (1997) describe estas variedades en detalle.

La parcela experimental fue de tres surcos de cinco metros de largo, a una densidad de siembra de 40,000 plantas ha-1. El manejo agronómico se realizó bajo las recomendaciones regionales (Parga, 1991).

El análisis estadístico se realizó en un diseño de bloques completos al azar, con cuatro repeticiones, con los diferentes arreglos (Normal, Parcelas Divididas o Parcelas Subdivididas, según los factores en estudio) y la diferencia de medias por la prueba de Tukey al 0,05 de probabilidad (Steel y Torrie, 1980).

El Análisis de componentes principales (ACP) se realizó con el paquete computacional Statistica.

\section{RESULTADOS Y DISCUSIÓN}

En el Cuadro 1, se presenta la matriz de correlación simple de las variables analizadas, en relación al rendimiento, así como todas las variables entre sí. De las variables morfológicas se encontró correlación alta con altura de planta y peso seco de hojas. Con las variables agroclimáticas no se encontró correlación significativa. De las variables fisiológicas, solo se encontró correlación significativa con contenido de clorofila, y esta, a su vez, tuvo la correlación más alta, entre todas las variables analizadas, con fotosíntesis. Crosbie y Pearce (1982), así como Mehta y Sarkar (1992) enfatizan la importancia de realizar las correlaciones con rendimiento desde el inicio de estudios de introducción de genotipos para siembra extensiva o para ser usados en programas de mejoramiento, puesto que seleccionar por alta o baja fotosíntesis, no asegura que hay mayor acumulación de fotosintetizados en los órganos de importancia económica.

Al realizar el ACP, en el Cuadro 2, se muestra que hasta el componente tres se explica el 79 por ciento de la varianza total. En el Cuadro 3 y Figuras 1, 2 y 3, se muestran las variables analizadas y su contribución relativa de los tres componentes principales, siendo las variables morfológicas las que más contribuyen a la variación en el componente 1 , y en el componente 2 , son las variables fisiológicas, permitiendo esta técnica precisar la contribución de las variables a los componentes principales y su relación con la varianza explicada (Ghawas, 1985). 


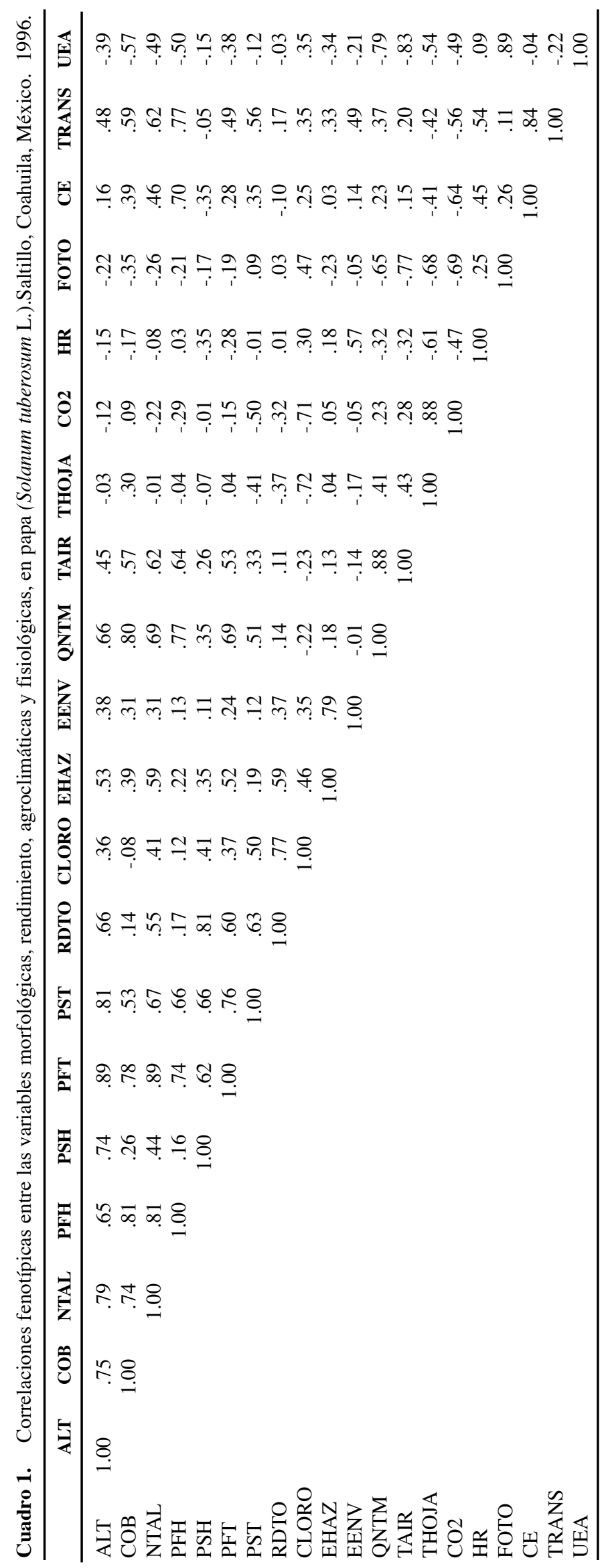


Cuadro 2. Análisis de componentes principales (Eigenvalores) entre variables morfológicas, climáticas, fisiológicas y rendimiento, en siete genotipos de papa (Solanum tuberosum L.). Saltillo, Coahuila, México. 1996.

\begin{tabular}{ccccc}
\hline Valor & Eigenvalor & $\begin{array}{c}\text { \% Varianza } \\
\text { Total }\end{array}$ & $\begin{array}{c}\text { Eigenval. } \\
\text { Acum. }\end{array}$ & $\begin{array}{c}\text { \% } \\
\text { Acumulado }\end{array}$ \\
\hline 1 & 7,87 & 39,36 & 7,87 & 39,36 \\
2 & 5,15 & 25,75 & 13,02 & 65,11 \\
3 & 2,80 & 14,0 & 15,82 & 79,12 \\
4 & 2,03 & 10,18 & 17,86 & 89,29 \\
5 & 0,87 & 4,37 & 18,73 & 93,66 \\
\hline
\end{tabular}

Cuadro 3. Contribución relativa de cada variable en los tres primeros componentes principales en papa (Solanum tuberosum L.). Saltillo, Coahuila, México. 1996.

\begin{tabular}{lccc}
\hline & \multicolumn{3}{c}{ Componentes Principales } \\
\cline { 2 - 4 } & $\mathbf{1}$ & $\mathbf{2}$ & $\mathbf{3}$ \\
\hline ALT & $0,90^{*}$ & 0,043 & 0,27 \\
COB & $0,83^{*}$ & $-0,24$ & $-0,24$ \\
NTAL & $0,94^{*}$ & 0,049 & $-0,021$ \\
PFH & $0,85^{*}$ & 0,013 & $-0,45$ \\
PSH & 0,55 & 0,013 & $0,76^{*}$ \\
PFT & $0,92^{*}$ & 0,008 & 0,18 \\
PST & $0,78^{*}$ & 0,34 & 0,14 \\
RDTO & 0,57 & 0,38 & $0,67 *$ \\
CLORO & 0,33 & $0,79^{*}$ & 0,37 \\
EHAZ & 0,56 & 0,11 & 0,30 \\
EENV & 0,37 & 0,30 & 0,05 \\
QNTM & $0,79^{*}$ & $-0,50$ & $-0,18$ \\
TAIR & 0,65 & $-0,58$ & $-0,16$ \\
THOJA & $-0,01$ & $-0,93^{*}$ & 0,025 \\
CO2 & $-0,17$ & $-0,89^{*}$ & 0,19 \\
HR & $-0,05$ & 0,60 & $-0,41$ \\
FOTO & $-0,34$ & $0,81^{*}$ & $-0,005$ \\
CE & 0,40 & 0,44 & $-0,75$ \\
TRANS & 0,66 & 0,45 & $-0,55$ \\
UEA & $-0,57$ & 0,65 & 0,18 \\
Expl.Var & 7,87 & 5,15 & 2,80 \\
Prp.Totl & 0,39 & 0,26 & 0,14 \\
\hline
\end{tabular}

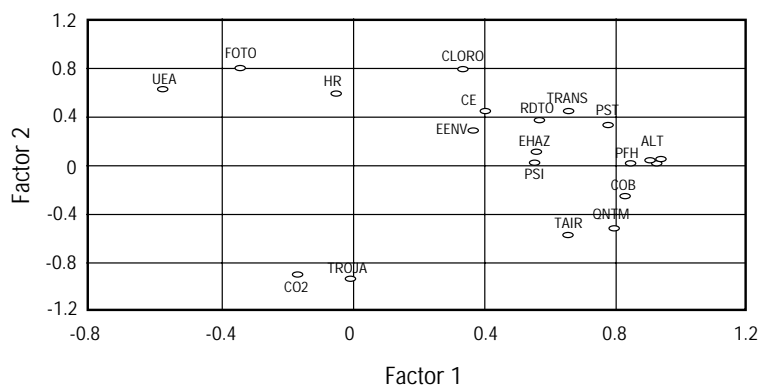

Figura 1. Posición de las variables analizadas, con los componentes 1 y 2 en papa (S. tuberosum L.). Saltillo, Coahuila, México. 1996.

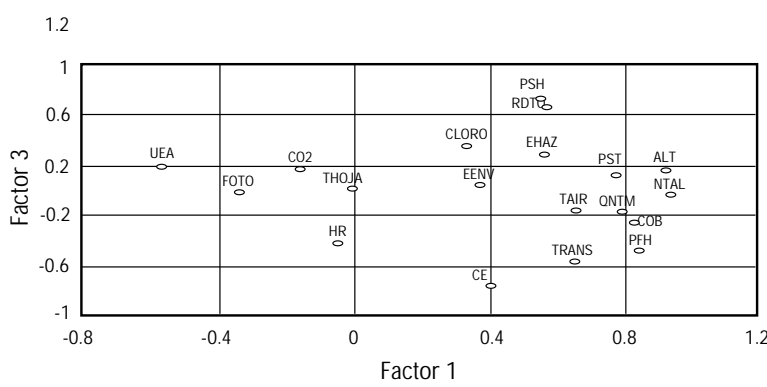

Figura 2. Posición de las variables analizadas, con los componentes 1 y 3 en papa ( $S$. tuberosum L.). Saltillo, Coahuila, México. 1996.

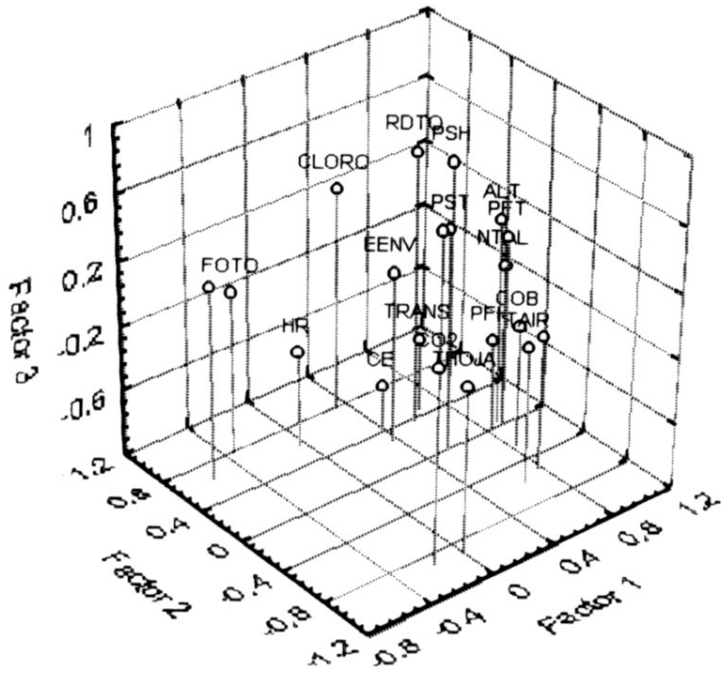

Figura 3. Posición de las variables analizadas, con los componentes 1, 2 y 3 en papa (S. tuberosum L.). Saltillo, Coahuila, México. 1996.

En el Cuadro 4, se presenta el comportamiento (positivo y negativo) de cada genotipo de papa para cada uno de los tres componentes principales, considerando

Cuadro 4. Comportamiento (positivo y negativo) de siete genotipos de papa (Solanum tuberosum L.) en invernadero para tres componentes principales. Saltillo, Coahuila, México. 1996.

\begin{tabular}{lrcr}
\hline & \multicolumn{3}{c}{ Componentes Principales } \\
\cline { 2 - 4 } Variedad & C 1 & C 2 & C 3 \\
\hline Norteña & 0,84 & 1,057 & 1,70 \\
Gigant & $-0,76$ & $-1,57$ & 0,19 \\
Mondial & 0,57 & 0,81 & $-1,38$ \\
Snowden & $-1,87$ & 1,04 & $-0,12$ \\
Alpha & $-0,08$ & $-0,69$ & 0,78 \\
Atlantic & 0,26 & $-0,005$ & $-0,71$ \\
R. Burbank & 1,03 & $-0,64$ & $-0,45$ \\
\hline
\end{tabular}


con mayor contribución las variables significativas (Judez, 1989). La variedad Norteña, presentó valores positivos en los tres componentes principales, siendo las variedades Gigant y Snowden los que presentan valores más negativos, como se observa en la Figura 4.

En la Figura 5, se presenta la posición de las variedades de papa, en función de los dos componentes principales, destacando las variedades Norteña, Mondial y Atlantic, con comportamiento positivo. Helachkar (1990) también encontró superioridad de la variedad Norteña, aunque evaluó atributos de rendimiento, morfológicos y de análisis de crecimiento. En la Figura 6, se presenta el comportamiento de la papa en algunas variables fisiológicas que no se habían estudiado en la región. Destaca Snowden, con mejor Uso Eficiente del Agua, pero con bajo rendimiento. La variedad Norteña, superior al testigo (Alpha), mostró el más alto rendimiento, aunque un Uso del Agua Intermedio, indicándonos en este caso, que puede presentar una relación fuente-demanda superior, con mayor tasa de acumulación de fotosintetizados en el tubérculo que las demás variedades, confirmando la importancia de realizar una evaluación integral, con la técnica ACP.

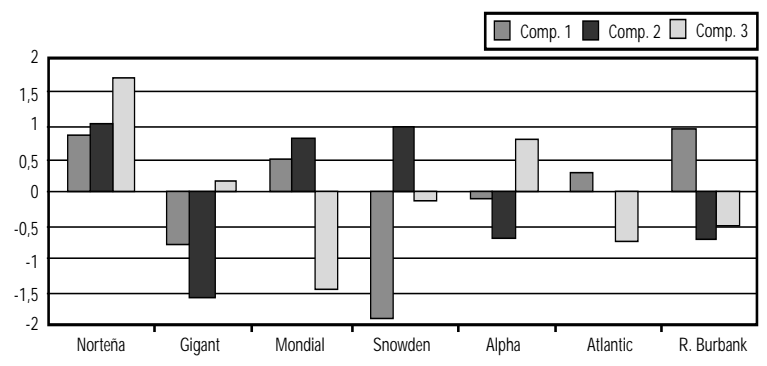

Figura 4. Comportamiento (Positivo y Negativo) para los tres componentes principales, de siete genotipos de papa (Solanum tuberosum L.) en campo. Saltillo, Coahuila, México. 1996.

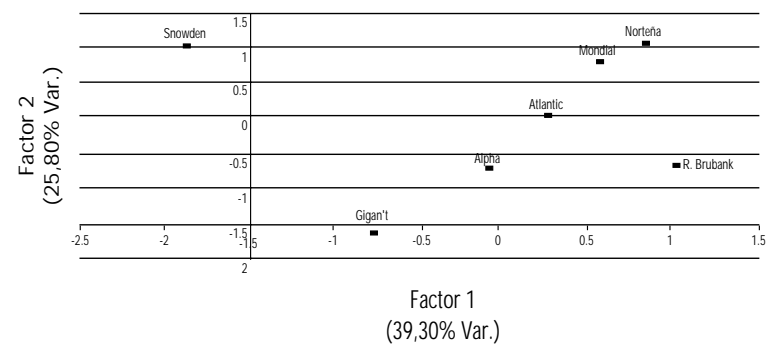

Figura 5. Posición de siete genotipos de papa (Solanum tuberosum L.) en campo, en función de los dos Componentes Principales en papa ( $S$. tuberosum L.). Saltillo, Coahuila, México. 1996.

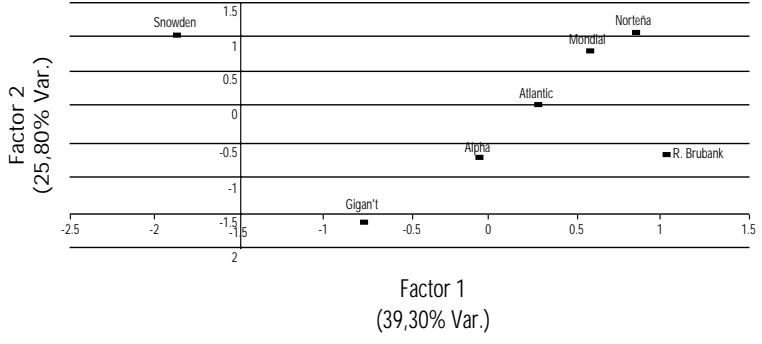

Figura 6. comportamiento de siete genotipos de papa (Solanum tuberosum L.) en las variables fotosíntesis $\left(\mu \mathrm{molCO} \mathrm{m}^{-2} \mathrm{~s}^{-1}\right)$, transpiración (mol $\mathrm{H}_{2} \mathrm{O} \mathrm{m} \mathrm{m}^{-2} \mathrm{~s}^{-1}$ ), Uso eficiente del agua y rendimiento $\left(\mathrm{kg} \mathrm{pta}^{-1}\right)$. Saltillo, Coahuila, México. 1996.

\section{CONCLUSIONES}

1. Existió alta correlación de algunas variables con el rendimiento en papa, siendo las más altas las de altura y peso seco de hojas. Estos son caracteres muy afectados por el ambiente. Las variables fisiológicas no presentaron correlación significativa.

2. Los componentes principales de variación en papa, fueron altura, cobertura, número de tallos y peso seco de hojas, en el componente uno, y fotosíntesis y contenido de clorofila en el componente dos.

3. La mejor variedad en todas las variables evaluadas en el análisis multivariado, fue la Norteña, seguida de la Mondial y Atlantic.

\section{LITERATURA CITADA}

CROSBIE, T.M.; PEARCE, R. 1982. Effects of recurrent phenotypic selection for high and low photosynthesis on agronomic traits in two maize populations. Crop Sci. Vol. 22:809-813.

FARÍAS, F.; THOMAS, N.; QUIROGA, H.M. 1983. Utilización del análisis de componentes principales en la selección de líneas y variedades introducidas de Ballico anual (Lolium multiflorum L.). Agricultura Técnica en México 9(2): 125-140.

GHAWAS, E.A. 1985. Analysis of components of plant yield variation in maize. Maize Abstracts 1(1):16.

GODSHALK, B.E.; TIMOTHY, H. 1988. Factor and 
principal component analysis as alternatives to index selection. Theor. Appl. Genet. 76:352-360.

HAY, R.K.M.; WALKER, A. 1989. An introduction to the physiology of crop yield. Longman Scientific \& Technical. New York. p.

HELACHKAR, J.S.H. 1990. Evaluación de genotipos de papa (Solanum tuberosum L.) por el método de análisis de componentes principales en diferentes localidades en el Sur de Coahuila y Nuevo León. Tesis Profesional, Universidad Autónoma Agraria "Antonio Narro", Saltillo, Coahuila, México. p.

JUDEZ, A.L. 1989. Técnicas de análisis de datos multidimen- sionales. Ministerio de Agricultura, Pesca y Alimentación. Madrid, España. p.

MEHTA, H.; SARKAR,K. 1992. Heterosis for leaf photosynthesis, grain yield and yield components in maize. Euphytica 60:161-168.

SOTO, G. 1997. Evaluación de variedades de papa (Solanum tuberosum L.) con criterios morfológicos, fisiológicos y de rendimiento. Tesis de Licenciatura. Universidad Autónoma Agraria “Antonio Narro". Buenavista, Saltillo, Coahuila. México. p.

STEEL, R.; TORRIE,J. 1980. Principles and procedures of statistics with special reference to the biological scien- 